\title{
Spotlight on Spironolactone Oral Suspension for the Treatment of Heart Failure: Focus on Patient Selection and Perspectives
}

This article was published in the following Dove Press journal:

Vascular Health and Risk Management

\author{
Antonis A Manolis' \\ Theodora A Manolis ${ }^{2}$ \\ Helen Melita ${ }^{3}$ \\ Antonis S Manolis (D) 4 \\ 'Patras University School of Medicine, \\ Patras, Greece; ${ }^{2}$ Red Cross Hospital, \\ Athens, Greece; ${ }^{3}$ Onassis Cardiac \\ Surgery Center, Athens, Greece; ${ }^{4}$ Athens \\ University School of Medicine, Athens, \\ Greece
}

\begin{abstract}
Spironolactone, an antagonist of aldosterone, initially used as a potassium-sparing diuretic, was subsequently shown to be a very effective adjunctive agent in the treatment of patients with heart failure with reduced ejection fraction, by halting the disease progression, with significant beneficial effects on both morbidity and mortality. Other uses comprise resistant hypertension, edema in patients with cirrhosis, and other on- and off-label uses. Recent data indicate that spironolactone also may offer some symptomatic relief in patients with heart failure and preserved ejection fraction. However, a variable percentage of patients, particularly among the aged group, may have difficulty in swallowing or may be unable to swallow tablets and thus are deprived of the benefits of such therapy. In 2017, the FDA approved a liquid suspension formulation of spironolactone, CaroSpir ${ }^{\circledR}$, which will enable more heart failure and other patients in need of aldosterone inhibition to avail themselves of the protective and beneficial effects of spironolactone. The new drug formulation comes as a banana-flavored oral suspension that contains $25 \mathrm{mg} / 5 \mathrm{~mL}$ of spironolactone, supplied in 4-ounce $(118 \mathrm{~mL})$ and 16-ounce $(473 \mathrm{~mL})$ bottles. The details of this drug formulation development and the benefits of spironolactone use in patients with heart failure with a focus on patient selection are herein reviewed.
\end{abstract}

Keywords: spironolactone, mineralocorticoid receptor antagonists, spironolactone oral suspension, heart failure, heart failure with reduced ejection fraction, hyperkalemia, ability to swallow

\section{Introduction}

Spironolactone is a mineralocorticoid receptor antagonist (MRA), specifically an antagonist of aldosterone, acting primarily through competitive binding of receptors at the aldosterone-dependent sodium-potassium exchange site in the distal convoluted renal tubule. Initially, spironolactone was considered and employed as a potassium-sparing diuretic, but it was subsequently shown to be a very effective adjunctive agent in the treatment of heart failure. ${ }^{1}$ Spironolactone has a primary role in managing patients with heart failure with reduced ejection fraction (HFrEF) by halting the disease progression, with significant beneficial effects on morbidity and mortality, across the spectrum of HFrEF, including patients after a myocardial infarction. ${ }^{2,3}$ Furthermore, spironolactone has an important role in patients with resistant hypertension. ${ }^{4}$ Importantly, there is also evidence from pivotal trials (RALES) that spironolactone, or MRAs in general, reduce the risk for sudden cardiac death (SCD) by $\sim 20 \%$ in patients with $\mathrm{HFrEF}$ and/or left ventricular dysfunction. ${ }^{5}$ Therapeutic concentrations of spironolactone block human
Correspondence: Antonis S Manolis Third Department of Cardiology, Ippokrateio Hospital, Vas. Sofias II4, Athens, 115 27, Greece

Tel $+30-2 / 3-2088470$

Fax +30-2/3-2088676

Email asm@otenet.gr 
ether-a-go-go-related gene (HERG) $\mathrm{K}+$ channels, and this may account for the antiarrhythmic effects of spironolactone in heart failure.

The main reservation for a more widespread use of spironolactone, particularly in men, relates to its progestational and anti-androgenic effects due to its non-specific binding to various steroid receptors; hence in men there may be a preference for use of eplerenone, another MRA agent almost devoid of these antiandrogenic effects. However, several studies have demonstrated a relatively superior efficacy of spironolactone over eplerenone and thus overall there may be a potential preference of spironolactone use in patients with heart failure. ${ }^{6,7} \mathrm{~A}$ recent study indicated that although gynecomastia in men was more common in the spironolactone vs the eplerenone group $(p=0.018)$, the discontinuation rates due to adverse events were similar in the two groups of heart failure patients receiving an MRA. ${ }^{8}$ Furthermore, spironolactone is much less expensive than eplerenone. ${ }^{7}$ Finally, there is some evidence from a recent meta-analysis demonstrating that the use of spironolactone improves left ventricular diastolic function in patients with heart failure with preserved ejection fraction (HFpEF), albeit without benefit on total mortality. ${ }^{9}$

Spironolactone has been available in the form of tablets under the trade name of Aldactone ${ }^{\circledR}$ (reference listed drug) and numerous other brand names. Only recently did the drug become available as an oral suspension under the tradename of CaroSpir ${ }^{\circledR}$. Apropos with this development, a review of this formulation of spironolactone is herein presented.

\section{Spironolactone Tablets}

As mentioned, spironolactone has been available as a tablet under various brand names worldwide. This standard tablet formulation of spironolactone comes in the form of oral tablets containing $25 \mathrm{mg}, 50 \mathrm{mg}$, or $100 \mathrm{mg}$ of the active drug. The drug was patented and marketed around 1960. Spironolactone is practically insoluble in water, soluble in alcohol, and freely soluble in benzene and in chloroform. Inactive ingredients include calcium sulfate, corn starch, flavor, hypromellose, iron oxide, magnesium stearate, polyethylene glycol, povidone, and titanium dioxide. The drug was first approved by the FDA in 1960; however, it gained its new indication for treating patients with HFrEF after the results of a landmark randomized controlled trial (RCT) (RALES trial) were published in 1999 (www.accessdata.fda.gov/scripts/cder/daf/ index.cfm?event $=$ overview.process \&ApplNo $=012151$ ). ${ }^{3}$
Spironolactone is absorbed partially ( $\sim 65 \%)$, is metabolized extensively (even during its first passage through the liver), undergoes enterohepatic recirculation, and is highly protein-bound. ${ }^{10}$ Spironolactone, after oral tablet intake, reaches a maximum concentration in $2.6 \mathrm{hrs}$ and an active metabolite (canrenone) reaches a maximum concentration in $4.3 \mathrm{hrs}$. When taken with food, its bioavailability increases to $\sim 95 \%$. Spironolactone has a half-life of $1.6 \mathrm{hrs}$, while its metabolite, canrenone, has a half-life of $16.5 \mathrm{hrs,}$ thus prolonging the biological effects of spironolactone.

\section{Oral Suspension of Spironolactone}

Carolina Medical Products Company, known as CMP Pharma, Inc. (Farmville, NC, US), developed an oral suspension of spironolactone, which is the first oral liquid form of spironolactone, intended for adults who have difficulty swallowing or who cannot swallow tablets. Prior to the approval of this formulation, patients had to obtain liquid spironolactone as a pharmacy compounded formulation, which commonly had limited shelf-life. Furthermore, an additional problem with these compounded liquid preparations is that they may include potentially harmful excipients. ${ }^{11}$ Other practices included tablet crushing and mixing it with fluids in order to facilitate swallowing with several drawbacks incurred by such alterations (see discussion below). ${ }^{12}$

After an application to the US Food and Drug Administration (FDA) by CMP Pharma, Inc., on August 4, 2017, the FDA approved spironolactone oral suspension $25 \mathrm{mg} / 5 \mathrm{~mL}$ ( $5 \mathrm{mg} / \mathrm{mL}$ ) for use in patients with heart failure, hypertension and edema caused by cirrhosis (www.accessdata. fda.gov/drugsatfda_docs/nda/2017/2094780rig1s000Approv. pdf) (Table 1). The following FDA statement was issued: "An alternative method of administration common in clinical practice for spironolactone tablets and its generic equivalents is compounding into an oral suspension".

The FDA approved the liquid drug formulation for the following indications (www.accessdata.fda.gov/drugsatfda docs/nda/2017/2094780rig1s000ChemR.pdf) (Table 1): 1) Heart failure; the FDA stated that the oral suspension of spironolactone is indicated for treatment of New York Hear Association (NYHA) class III-IV HFrEF to increase survival, manage edema, and to reduce the need for hospitalization for heart failure; 2) Hypertension; spironolactone oral suspension is indicated as add-on therapy for the treatment of hypertension, to lower blood pressure in adult patients who are not adequately controlled on other agents; 3) Edema caused by cirrhosis; spironolactone oral suspension is 
Table I FDA Approved Indications for the Oral Liquid Suspension of Spironolactone

\begin{tabular}{|l|l|}
\hline Indication & Aim \\
\hline $\begin{array}{l}\text { - Heart failure with reduced ejection fraction, NYHA } \\
\text { class III-IV }\end{array}$ & $\begin{array}{l}\text { To increase survival, manage edema, and to reduce the need for hospitalization for } \\
\text { heart failure }\end{array}$ \\
\hline$\bullet$ Hypertension as add-on therapy & To lower blood pressure in adult patients who are not adequately controlled on other agents \\
\hline$\bullet$ Edema caused by cirrhosis & To manage edema that is not responsive to fluid and sodium restriction \\
\hline
\end{tabular}

Notes: N.B.: Patients requiring a dose $>100 \mathrm{mg}$ should not use spironolactone oral suspension $(25 \mathrm{mg} / 5 \mathrm{~mL})$ as this is not therapeutically equivalent to spironolactone tablets; doses of the suspension $>100 \mathrm{mg}$ may result in spironolactone concentrations higher than expected.

Abbreviations: FDA, Food and Drug Administration; NYHA, New York Heart Association.

indicated for the management of edema in adult cirrhotic patients when edema is not responsive to fluid and sodium restriction.

As mentioned, the oral suspension of spironolactone contains $25 \mathrm{mg} / 5 \mathrm{~mL}$ of spironolactone. The drug product is a white to off-white, opaque, banana-flavored, immediate release oral suspension. The product is supplied in 4-ounce $(118 \mathrm{~mL})$ and 16 -ounce $(473 \mathrm{~mL})$ bottles. Inactive ingredients include xanthan gum, simethicone emulsion, sorbic acid, potassium sorbate, sodium saccharin, citric acid anhydrous, sodium citrate dihydrate, Magnasweet 110, glycerin, banana flavor, and purified water.

According to the FDA documents (www.accessdata.fda. gov/drugsatfda docs/nda/2017/2094780rig1s000ChemR. pdf), the manufacturer (CMP Pharma) conducted two bioavailability studies, comparing spironolactone oral suspension to the reference listed drug (RLD) of spironolactone tablet. One study was conducted comparing a 25 -mg spironolactone tablet to spironolactone oral suspension (Study 063-15), and another comparing 100-mg tablets to spironolactone oral suspension (Study 064-15). In addition, a food-effect study was conducted on spironolactone oral suspension at $100 \mathrm{mg}$ (Study 084-15). The results showed that the bioavailability of the spironolactone suspension is approximately $15 \%$ higher compared to spironolactone 25 -mg tablets and $\sim 35 \%$ higher compared to spironolactone 100-mg tablets. To address the higher exposures, the FDA proposed a dose adjustment between $25 \mathrm{mg}$ and $100 \mathrm{mg}$.

As discussed in the Office of Clinical Pharmacology (OCP) review of the FDA, the relative bioavailability studies indicate that the bioavailability of spironolactone suspension is $15 \%$ and $37 \%$ higher than the RLD at the $25-\mathrm{mg}$ and $100-$ $\mathrm{mg}$ tablet strengths, respectively. The food-effect study indicates that food has a significant impact on the bioavailability of spironolactone suspension, as is also true for the RLD. After administration of $100 \mathrm{mg}$ of the spironolactone suspension with a high-fat and high-calorie meal, the Cmax decreased by $22 \%$ and the area under the curve (AUC) increased by $90 \%$ and Tmax was delayed from $0.9 \pm$ $0.3 \mathrm{hrs}$ to $2.4 \pm 1.0 \mathrm{hrs}$ in the fasted and fed state, respectively.

Given the findings in the relative bioavailability studies (i.e., the different relative bioavailability estimates obtained in the comparison against the $25-$ and $100 \mathrm{mg}$ tablet strengths) and the lack of information on dose proportionality for spironolactone tablets, the review team could not exclude the possibility that doses of the suspension $>100 \mathrm{mg}$ could result in spironolactone concentrations higher than expected relative to the RLD. The review team recommended a $25 \%$ dose reduction to adjust for the higher exposure to spironolactone from the suspension between the dose range of 25 and $100 \mathrm{mg}$.

According to the manufacturer (www.carospir.com/pre scribing-information/), spironolactone oral suspension is a stable, ready to use, and consistent liquid treatment option for adult patients who have difficulty swallowing, or who cannot swallow tablets. Spironolactone oral suspension is not therapeutically equivalent to spironolactone tablets. For an equivalent dose, spironolactone oral suspension results in 15 to $37 \%$ higher serum concentration compared to spironolactone tablets. A high fat and high calorie meal increased the bioavailability of spironolactone by approximately $90 \%$. Patients should establish a routine pattern for taking spironolactone oral suspension with regard to meals.

As alluded to above, spironolactone oral suspension ( $25 \mathrm{mg} / 5 \mathrm{~mL}$ ) is not therapeutically equivalent to spironolactone tablets. Doses of the suspension $>100 \mathrm{mg}$ may result in spironolactone concentrations higher than expected. Thus, another formulation should be used in patients requiring a dose $>100 \mathrm{mg}$.

According to the quality assessment of the available stability data, the expiry dating was set at 18 months for the 4-ounce bottle and at 24 months for the 16-ounce bottle. The 
oral suspension is stored at $20^{\circ} \mathrm{C}$ to $25^{\circ} \mathrm{C}\left(68^{\circ} \mathrm{F}\right.$ to $\left.77^{\circ} \mathrm{F}\right)$, with excursions permitted to $15^{\circ} \mathrm{C}$ to $30^{\circ} \mathrm{C}\left(59^{\circ} \mathrm{F}\right.$ to $\left.86^{\circ} \mathrm{F}\right)$.

\section{Side-Effects}

Spironolactone can cause side effects, such as fluid and electrolyte imbalance (hyperkalemia, hyponatremia), mild acidosis, and transient elevation of serum urea nitrogen. Importantly, hyperkalemia can be fatal and patient's potassium level needs to be checked while on spironolactone. As an anti-androgenic agent, it may produce loss of libido, gynecomastia/mastodynia and menstrual irregularities.

\section{Carcinogenesis}

Across 5 studies examining spironolactone only (not in combination with other agents) there was no indication that spironolactone increased the risk of any of the cancers examined (www.accessdata.fda.gov/drugsatfda_docs/nda/ 2017/2094780rig1s000SumR.pdf). One of the largest studies, conducted in the UK Clinical Practice Research Database (CPRD, examining 36 cancers), suggested that spironolactone might actually be associated with a reduced risk of prostate cancer. Among the three case-control studies examining spironolactone grouped with diuretics in a similar class, there were some suggestions of an increased risk of breast cancer and squamous cell carcinoma, although limitations including recall bias and missing information on important lifestyle factors reduces the credibility and validity of these findings. The increased cancer risks found may also be attributable to the other drugs and not just to spironolactone. Overall there is little evidence that spironolactone is associated with an increased risk of any cancer.

\section{Pediatrics}

Due to safety concerns, the drug was not approved for pediatric use in hypertension or heart failure. Hypertension requires chronic treatment and there are concerns for spironolactone use in children due to the drug's anti-androgenic, progestogenic, and estrogenic properties. Also, heart failure causes and mechanisms in children are different from those in adults. Heart failure in children is mainly due to congenital heart disease or cardiomyopathy, whereas in adults heart failure is mainly due to ischemic heart disease.

\section{Drug Interaction}

The label states that spironolactone and its metabolites interfere with radioimmunoassays for digoxin and increase the apparent exposure to digoxin while it is unknown to what extent, if any, spironolactone may increase actual digoxin exposure. Use of a digoxin assay is advised that does not interact with spironolactone.

\section{Pregnancy and Lactation}

Based on mechanism of action and findings in animal studies, spironolactone may affect sex differentiation of the male during embryogenesis (www.accessdata.fda.gov/ drugsatfda docs/nda/2017/2094780rig1s000SumR.pdf).

Rat embryofetal studies report feminization of male fetuses and endocrine dysfunction in females exposed to spironolactone in utero. Limited available data from published case reports and case series did not demonstrate an association of major malformations or other adverse pregnancy outcomes with spironolactone. Because of the potential risk to the male fetus due to anti-androgenic properties of spironolactone and animal data, spironolactone should be avoided during pregnancy or a pregnant woman should be advised of the potential risk to a male fetus.

\section{Hyperkalemia}

The major potential problem with the use of spironolactone relates to hyperkalemia, particularly in patients with renal dysfunction or patients receiving angiotensin converting enzyme inhibitors (ACEI) or angiotensin receptor blockers (ARBs), which may also elevate serum potassium levels. However, recently with the availability of a new agent in oral suspension, patiromer, a potassium binding polymer, the use of spironolactone is facilitated, since it can be coadministered with this agent and thus reduce the possibility of inducing hyperkalemia. Patiromer is a non-absorbed, sodium-free potassium-binding polymer that exchanges calcium for potassium in the gastrointestinal tract, thereby increasing fecal potassium excretion and reducing serum potassium levels. ${ }^{13}$ Oral patiromer is an important treatment option for the long-term management and/or prevention of hyperkalemia, particularly in patients receiving potassiumretaining agents, like spironolactone. A recent study of 295 patients with chronic kidney disease and resistant hypertension who were randomized to spironolactone in addition to double-blind treatment with either placebo $(n=148)$ or patiromer $(n=147)$, indicated that at week $12,66 \%$ of patients in the placebo group and $86 \%$ of patients in the patiromer group remained on spironolactone (between-group difference $19.5 \% ; \mathrm{p}<0.0001) .{ }^{14}$ Adverse events were mostly mild or moderate in severity and occurred in $53 \%$ vs $56 \%$ of patients in the two groups. The authors concluded that 
patiromer enabled more patients to continue treatment with spironolactone with less hyperkalemia.

A cost-effectiveness analysis of treatment with patiromer, spironolactone, and an ACEI in patients with New York Heart Association (NYHA) class III-IV heart failure deriving data from the RALES (spironolactone RCT) and OPAL-HK (patiromer RCT), indicated that treatment with patiromer-spironolactone-ACEI was projected to increase longevity compared with ACEI alone (5.29 vs 4.62 life-years gained, respectively), greater quality-adjusted life-years (QALYs) (2.79 vs 2.60), and costs (US\$28,200 vs US\$18,200), giving an incremental costeffectiveness ratio (ICER) of US\$52,700 per QALY gained. ${ }^{15}$ The authors concluded that the use of spironolactone-patiromer-ACEI may provide clinical benefit and good economic value in patients with NYHA class III-IV heart failure unable to tolerate spironolactone due to hyperkalemia.

Finally, it should be noted that despite concerns about MRA-induced hyperkalemia, a meta-analysis of 7 RCTs reporting on hyperkalemia in 16,065 patients after a myocardial infarction or with chronic heart failure, indicated that truly MRA-related hyperkalemia was 54\%, whereas $46 \%$ were non-MRA related. Specifically, in spironolactone trials, hyperkalemia was documented in $17.5 \%$ and in $7.5 \%$ of patients on placebo $(\mathrm{P}=0.0001){ }^{16}$ The authors concluded that non-MRA-related rises in potassium levels might be underestimated and should be rigorously explored before cessation of the evidence-based therapy with MRAs.

\section{Other Side-Effects/Drug Interactions}

As mentioned, the progestational and anti-androgenic effects of spironolactone are of concern particularly in men manifesting as decreased libido, impotence, gynecomastia, and mastodynia, while women may exhibit menstrual irregularities.

Salicylates may reduce the tubular secretion and decrease the diuretic efficacy of spironolactone. Spironolactone may alter the clearance of digitalis. Spironolactone also may induce diarrhea, gastritis, gastric bleeding, and peptic ulcers; the drug is contraindicated in patients with peptic ulcer disease. Central nervous system adverse effects include drowsiness, lethargy, ataxia, confusion, and headache. Spironolactone may cause rashes and, rarely, blood dyscrasias. As mentioned, whether therapeutic doses of spironolactone can induce malignancies remains doubtful.

According to the results of a systematic review (72 articles) and meta-analysis (12 RCTs), spironolactone may induce disease-specific and modest changes in glycemia; however, data from the most extensively studied population, individuals with diabetes, did not support a long-term glycemic impact in these patients. ${ }^{17}$

\section{Patient Selection}

As based on the pivotal trial, RALES, published in 1999, patients with HFrEF are the best candidates for treatment with spironolactone (class I indication, level of evidence A) (Table 2). ${ }^{3,18}$ RALES included 1663 patients with severe heart failure (NYHA class III/IV) and a left ventricular ejection fraction (LVEF) of $\leq 35 \%$ who were being treated with an ACEI, a loop diuretic, and in most cases digoxin, and randomized to receive $25 \mathrm{mg}$ of spironolactone daily $(\mathrm{n}=822)$ or placebo $(n=841) .^{3}$ The trial was discontinued early (at 24 months) due to efficacy of spironolactone. The mortality rate was $46 \%$ in the placebo group and $35 \%$ in the spironolactone group (relative risk of death, $0.70 ; \mathrm{P}<0.001$ ). This $30 \%$ reduction in the risk of death among patients in the spironolactone group was attributed to a lower risk of both death from progressive heart failure and SCD. The frequency of hospitalization for worsening heart failure was 35\% lower in the spironolactone group than in the placebo group (relative risk of hospitalization, 0.65 ; $\mathrm{P}<0.001$ ). In addition, patients who received spironolactone had a significant improvement in the symptoms of heart failure, as assessed on the basis of the NYHA functional class $(\mathrm{P}<0.001)$. The incidence of serious hyperkalemia was minimal in both groups of patients ( $2 \%$ vs $1 \%$ ). Gynecomastia or breast pain was reported by $10 \%$ of the men in the spironolactone group and $1 \%$ of the men in the placebo group $(\mathrm{P}<0.001)$, causing more patients in the spironolactone group than in the placebo group to discontinue treatment ( 10 vs $1, \mathrm{P}=0.006$ ). The authors concluded that blockade of aldosterone receptors by spironolactone, in addition to standard therapy, substantially reduces the risk of both morbidity and death among patients with severe heart failure.

A large RCT (TOPCAT) investigating the effect of spironolactone in 3445 patients with HFpEF indicated that spironolactone had a beneficial effect in only one of the components of the primary outcome; only hospitalization for HF was significantly lower in the spironolactone group than in the placebo group (12\% vs $14.2 \%$; hazard ratio-HR $0.83 ; \mathrm{P}=0.04) .{ }^{19}$ Neither total deaths nor 
Table 2 Patient Selection for Use of Liquid Suspension of Spironolactone in Patients with Heart Failure

\begin{tabular}{|c|c|c|c|}
\hline Patients & $\begin{array}{l}\text { Class of } \\
\text { Recommendation/ } \\
\text { LOE }\end{array}$ & Dose & Comments \\
\hline $\begin{array}{l}\text { - Patients with HFrEF, NYHA } \\
\text { class II-IV }\end{array}$ & $\mathrm{I} / \mathrm{A}$ & $\begin{array}{l}\text { Initial dose: } 12.5-25 \mathrm{mg} \\
\text { qd; maximal dose } 25 \mathrm{mg} \\
\text { qd }-25 \mathrm{mg} \text { bid }\end{array}$ & \\
\hline - Patients with HFpEF & $\mathrm{Ilb} / \mathrm{B}$ & Same & $\begin{array}{l}\text { Additional selection criteria: } \mathrm{LVEF} \geq 45 \% \text {, elevated BNP } \\
\text { levels or } \mathrm{HF} \text { admission within I year, eGFR }>30 \mathrm{~mL} / \mathrm{min} \\
\text { creatinine }<2.5 \mathrm{mg} / \mathrm{dL} \text {, potassium }<5.0 \mathrm{mEq} / \mathrm{L}\end{array}$ \\
\hline $\begin{array}{l}\text { - Candidate patients with } \\
\text { difficulty in swallowing or unable } \\
\text { to swallow tablet drug forms }\end{array}$ & & Same & \\
\hline $\begin{array}{l}\text { - Candidate patient preference/ } \\
\text { acceptability }\end{array}$ & & Same & Ease of swallowing, drug flavor/taste \\
\hline
\end{tabular}

Abbreviations: BNP, brain natriuretic peptide; eGFR, estimated glomerular filtration rate; HFpEF, heart failure with preserved ejection fraction; HFrEF, heart failure with reduced ejection fraction; LOE, level of evidence; LVEF, left ventricular ejection fraction; NYHA, New York Heart Association.

hospitalizations for any reason were significantly reduced by spironolactone. However, despite conflicting reports on the effect of spironolactone in this group of patients (HFpEF), a recent meta-analysis of 11 RCTs comprising 4539 patients indicated that spironolactone reduced hospitalizations (odds ratio - OR 0.84; $\mathrm{P}=$ 0.006), improved NYHA functional classifications (OR $0.35 ; \mathrm{P}=0.001$ ), and decreased the levels of brain natriuretic peptide (BNP) (mean difference - MD,$44.80 \mathrm{pg} / \mathrm{mL} ; \mathrm{P}=0.002$ ) in patients with heart failure with mid-range ejection fraction (HFmrEF) and patients with HFpEF. $^{20}$ The risks of hyperkalemia $(\mathrm{P}<0.001)$ and gynecomastia $(\mathrm{P}<0.001)$ were increased. The authors concluded that patients with HFmrEF and HFpEF could benefit from spironolactone treatment, albeit with increased risks of hyperkalemia and gynecomastia.

Based on the results of TOPCAT, current guidelines recommend that an aldosterone receptor antagonist may be considered in appropriately selected patients with HFpEF (with $\mathrm{LVEF} \geq 45 \%$, elevated BNP levels or heart failure admission within 1 year, estimated glomerular filtration rate $>30 \mathrm{~mL} / \mathrm{min}$, creatinine $<2.5 \mathrm{mg} / \mathrm{dL}$, potassium $<5.0 \mathrm{mEq} / \mathrm{L}$ ) (indication class IIb, level of evidence B) (Table 2). ${ }^{21}$

As mentioned, there is evidence that spironolactone or eplerenone reduce the risk for SCD by $\sim 20 \%$ in patients with HFrEF and/or left ventricular dysfunction. ${ }^{5}$ This favorable effect seems to be related to an improvement in cardiac fibrosis, and potentially, the prevention of hypokalemia-related ventricular tachyarrhythmias. ${ }^{22}$
In patients with milder heart failure (NYHA I-II), it seems that the benefit of spironolactone treatment is attenuated. $^{23}$ Hence, the best outcome is observed in patients with heart failure symptoms NYHA class III-IV, as demonstrated in the RALES trial, ${ }^{3}$ however, current guidelines indicate that patients with symptomatic HFrEF (NYHA class II-IV, LVEF $\leq 35 \%$ ) who remain symptomatic despite treatment with an ACEI or ARB and a betablocker, should receive an MRA to reduce the risk of heart failure hospitalization and death (class I, level of evidence A) (Table 2). ${ }^{18}$ Caution should be exercised in patients with impaired renal function and in those with serum potassium levels $>5.0 \mathrm{mmol} / \mathrm{L}$ (Box 1); regular checks of serum potassium levels and renal function should be performed according to clinical status.

Patients with end-stage renal disease is generally a difficult to treat group of patients. The use of MRAs in these patients is hindered by concerns that their cardioprotective actions may be counteracted by excess risk of hyperkalemia. A systematic review indicated that a growing body of evidence derived from small RCTs suggests that MRA therapy improves a number of surrogate cardiovascular risk

\section{Box I Contra-Indications of Spironolactone Use}

- Severe renal dysfunction/end-stage renal disease *

- $\mathrm{K}+$ level $>5 \mathrm{mE} / \mathrm{L} *$

- Addison's disease

Note: *With the availability of patiromer, a potassium binding polymer, it may be feasible in the future to circumvent this limitation 
factors in long-term dialysis patients, translating into a clinically relevant (up to 60\%) reduction in the risk of allcause and cardiovascular mortality. ${ }^{24}$ However, MRA use was shown to be accompanied by a parallel increase in the risk of hyperkalemia and gynecomastia, thus currently prohibiting the wide use of MRAs in dialysis patients. Whether the combined use of spironolactone and patiromer (that counteracts hyperkalemia) may change this prospect remains to be seen.

Importantly, now with the availability of the oral suspension of spironolactone, more heart failure patients can avail themselves of the benefits of this MRA agent, who are not able to swallow or have great difficulty in swallowing tablets.

The beneficial effects of spironolactone on mortality in patients with HFrEF appear to be additive to those of ACEI/ARBs and $\beta$-blockers. Caution should be exercised in patients with significant renal dysfunction for fear of hyperkalemia (Box 1). Treatment should be initiated at a low dose (12.5-25 mg daily), which is greatly facilitated with the availability of the oral suspension formulation containing $25 \mathrm{mg} / 5 \mathrm{~mL}(5 \mathrm{mg} / \mathrm{mL})$, as higher doses may lead to hyperkalemia, particularly in patients receiving an ACEI or an ARB. Serum K+ levels should be checked after initiation of treatment, and vigilance is recommended for potential drug interactions and medical disorders that may elevate serum $\mathrm{K}+$ concentration (e.g., potassium supplements, ACEI/ARBs, and worsening renal function).

\section{Tablet versus Liquid Formulation Patient Acceptability/Ability to Swallow}

The ability to swallow may determine patient acceptability of conventional tablet formulations of drugs. The natural process of aging is associated with a decline in swallowing function. Difficulties in swallowing and esophageal retention of tablets and capsules are expected to be magnified in older adults. Data suggest that about one third of the older patients in long-term care facilities experience swallowing problems with solid oral drug products, ${ }^{25}$ community data raise this percentage to $60 \%{ }^{26}$ The prevalence for developing swallowing dysfunctions is significantly higher in central nervous or neuromuscular disease conditions like Parkinson's disease, Alzheimer's disease, stroke, amyotrophic lateral sclerosis or motor neuron disease. ${ }^{27}$ However, symptoms of dysphagia are experienced by all age groups and are not solely limited to the older and multi-morbid patient.

\section{Formulation Design/Available Options}

Formulation design and selection affect the acceptability of medicines. Characteristics of a medicine affecting safe swallowing are of significant importance in older patients, especially to prevent esophageal retention and risk of aspiration. ${ }^{27}$ Caregivers and family members and/or patients themselves frequently resort to crushing tablets or opening capsules in order to circumvent the difficulty in swallowing solid drugs. However, such drug alteration implies a high risk for potential drug instability and modification of the product performance that is neither tested nor authorized by the pharmaceutical manufacturer and the regulatory bodies. Such alterations may lead to serious adverse effects or severe intoxication of the patients, ${ }^{12}$ or may produce a bad taste of the ingredients. Hence, the availability of a drug in liquid suspension formulation may circumvent such obstacles in drug intake. As mentioned, spironolactone oral suspension comes in a white to off-white, opaque, banana-flavored, immediate release oral suspension, which can facilitate oral intake of spironolactone.

\section{Conclusion}

Spironolactone has an established benefit in patients with heart failure, particularly patients with HFrEF, according with the results of the pivotal RALES trial. However, a percentage of patients who could not swallow or had difficulty in swallowing could not avail themselves of these drug benefits. Now with the availability of the oral suspension formulation of the drug, they also can take advantage of this useful treatment. Spironolactone therapy in this group of patients not only improves symptoms and left ventricular function, but most importantly prolongs survival. In the other group of patients with HFpEF, spironolactone has advantages in improving symptoms and left ventricular function, however, without reducing mortality. Caution is advised for the risk of hyperkalemia in patients receiving spironolactone, however, with the recent availability of another drug in oral suspension, patiromer, which counteracts this side-effect and reduces the risk of hyperkalemia, more patients can take advantage of spironolactone therapy.

\section{Perspective}

Although newer MRAs have become available or are emerging, they have not been shown to supersede spironolactone in efficacy. With the recent availability of the oral suspension of spironolactone, more patients can avail 
themselves of the highly beneficial effects of the drug in reducing morbidity and mortality in patients with heart failure, particularly those with HFrEF.

Current recommendations for the treatment of patients with HFrEF suggest the combined use of $\beta$-blockers, MRAs, angiotensin receptor neprilysin inhibitors, and cardiac resynchronization therapy. The cumulative benefits on SCD with the use of combination therapy are estimated to be additive, but difficult to discern individual drug contributions. $^{22}$ Nevertheless, it is very encouraging that the annual rate of SCD has been reduced by $45 \%$ to $60 \%$ over a period of 10 to 15 years in patients with HFrEF who were receiving real-world combination treatments, and this benefit was independent of the use of an implantable cardioverter defibrillator. $^{28}$

In the long run, personalized medicine is going to gain ground in the future, where a tailored approach to each patient could be offered in order to provide the most effective therapy, while reducing risks and adverse effects. $^{29}$ Currently, MRAs, with spironolactone as the major and most effective representative agent, need to be provided to the great majority of, if not all, patients with HFrEF in the absence of contra-indications due to compelling results indicating both symptomatic improvement and prolonged survival. Whether patients with HFpEF may have a consistent benefit for symptomatic improvement with use of MRAs has not been entirely settled, as yet; nevertheless, spironolactone should be considered for symptomatic HFpEF patients with elevated natriuretic peptides. Future trials targeting more specific groups of heart failure patients (e.g. severe renal impairment or elevated prespecified biomarkers, etc) may render possible a truly more personalized approach.

Again, with the availability of the liquid formulation of spironolactone, more patients can take advantage of the MRA benefits and participate in such trials exploring individually tailored and more personalized therapeutic strategies. Finally, with the availability of another agent, patiromer, as an oral suspension that can effectively counteract hyperkalemia which is the major potential complication and limitation of spironolactone therapy, the prospect of combining these two agents may facilitate and expand the use of spironolactone to a wider group of candidate patients.

\section{Abbreviations}

ACEI, angiotensin converting enzyme inhibitor; ARB, angiotensin receptor blocker; FDA, Food and Drug
Administration; HFpEF, heart failure with preserved ejection fraction; HFrEF, heart failure with reduced ejection fraction; LVEF, left ventricular ejection fraction; MRA, mineralocorticoid receptor antagonist; NYHA, New York Heart Association; RCT, randomized controlled trial; RLD, reference listed drug; SCD, sudden cardiac death.

\section{Disclosure}

The authors report no conflicts of interest in this work.

\section{References}

1. Lainscak M, Pelliccia F, Rosano G, et al. Safety profile of mineralocorticoid receptor antagonists: spironolactone and eplerenone. Int J Cardiol. 2015;200:25-29. doi:10.1016/j.ijcard.2015.05.127

2. Papademetriou V, Toumpourleka M, Imprialos KP, Alataki S, Manafis A, Stavropoulos K. The role of mineralocorticoid receptor antagonists in heart failure with reduced ejection fraction. Curr Pharm Des. 2018;24 (46):5517-5524. doi:10.2174/1381612825666190219141326

3. Pitt B, Zannad F, Remme WJ, et al. The effect of spironolactone on morbidity and mortality in patients with severe heart failure. Randomized Aldactone Evaluation Study Investigators. $N$ Engl J Med. 1999;341(10):709-717. doi:10.1056/NEJM199909023411001

4. Manolis AA, Manolis TA, Melita H, Manolis AS. Eplerenone versus spironolactone in resistant hypertension: an efficacy and/or cost or just a men's issue? Curr Hypertens Rep. 2019;21(3):22. doi:10.1007/ s11906-019-0924-0

5. Rossello X, Ariti C, Pocock SJ, et al. Impact of mineralocorticoid receptor antagonists on the risk of sudden cardiac death in patients with heart failure and left-ventricular systolic dysfunction: an individual patient-level meta-analysis of three randomized-controlled trials. Clin Res Cardiol. 2019;108(5):477-486. doi:10.1007/s00392-018-1378-0

6. Frankenstein L, Seide S, Tager $T$, et al. Relative Efficacy of Spironolactone, Eplerenone, and cAnRenone in patients with Chronic Heart failure (RESEARCH): a systematic review and network meta-analysis of randomized controlled trials. Heart Fail Rev. 2019. doi:10.1007/s10741-019-09832-y

7. Chatterjee S, Moeller C, Shah N, et al. Eplerenone is not superior to older and less expensive aldosterone antagonists. Am J Med. 2012;125(8):817-825. doi:10.1016/j.amjmed.2011.12.018

8. Yamamoto M, Seo Y, Ishizu T, et al. Comparison of effects of aldosterone receptor antagonists spironolactone and eplerenone on cardiovascular outcomes and safety in patients with acute decompensated heart failure. Heart Vessels. 2019;34(2):279-289. doi:10.1007/ s00380-018-1250-1

9. Li S, Zhang X, Dong M, et al. Effects of spironolactone in heart failure with preserved ejection fraction: a meta-analysis of randomized controlled trials. Medicine (Baltimore). 2018;97(35):e11942. doi:10.1097/MD.0000000000011942

10. Brunton L, Parker K, Blumenthal D, Buxton I. Goodman and Gilman's Manual of Pharmacology and Therapeutics. USA: McGraw-Hill Companies, Inc; 2008.

11. Binson G, Beuzit K, Migeot V, et al. Preparation and physicochemical stability of liquid oral dosage forms free of potentially harmful excipient designed for pediatric patients. Pharmaceutics. 2019;11:4. doi: $10.3390 /$ pharmaceutics 11040190

12. Cornish P. "Avoid the crush": hazards of medication administration in patients with dysphagia or a feeding tube. CMAJ. 2005;172 (7):871-872. doi:10.1503/cmaj.050176

13. Blair HA. Patiromer: a review in hyperkalaemia. Clin Drug Investig. 2018;38(8):785-794. doi:10.1007/s40261-018-0675-8 
14. Agarwal R, Rossignol P, Romero A, et al. Patiromer versus placebo to enable spironolactone use in patients with resistant hypertension and chronic kidney disease (AMBER): a Phase 2, randomised, double-blind, placebo-controlled trial. Lancet. 2019;394(10208):1540-1550. doi:10. 1016/S0140-6736(19)32135-X

15. Bounthavong M, Butler J, Dolan CM, et al. Cost-effectiveness analysis of patiromer and spironolactone therapy in heart failure patients with hyperkalemia. Pharmacoeconomics. 2018;36(12):1463-1473. doi:10.1007/s40273-018-0709-3

16. Vukadinovic D, Lavall D, Vukadinovic AN, Pitt B, Wagenpfeil S, Bohm M. True rate of mineralocorticoid receptor antagonists-related hyperkalemia in placebo-controlled trials: a meta-analysis. Am Heart J. 2017;188:99-108. doi:10.1016/j.ahj.2017.03.011

17. Korol S, Mottet F, Perreault S, Baker WL, White M, de Denus S. A systematic review and meta-analysis of the impact of mineralocorticoid receptor antagonists on glucose homeostasis. Medicine (Baltimore). 2017;96(48):e8719. doi:10.1097/MD.0000000000008719

18. Ponikowski P, Voors AA, Anker SD, et al. 2016 ESC Guidelines for the diagnosis and treatment of acute and chronic heart failure: the Task Force for the diagnosis and treatment of acute and chronic heart failure of the European Society of Cardiology (ESC) developed with the special contribution of the Heart Failure Association (HFA) of the ESC. Eur Heart J. 2016;37(27):2129-2200. doi:10.1093/eurheartj/ehw128

19. Pitt B, Pfeffer MA, Assmann SF, et al. Spironolactone for heart failure with preserved ejection fraction. $N$ Engl J Med. 2014;370 (15):1383-1392. doi:10.1056/NEJMoa1313731

20. Xiang Y, Shi W, Li Z, et al. Efficacy and safety of spironolactone in the heart failure with mid-range ejection fraction and heart failure with preserved ejection fraction: a meta-analysis of randomized clinical trials. Medicine (Baltimore). 2019;98(13):e14967. doi:10.1097/ MD.0000000000014967

21. Yancy CW, Jessup M, Bozkurt B, et al. 2017 ACC/AHA/HFSA focused update of the $2013 \mathrm{ACCF} / \mathrm{AHA}$ guideline for the management of heart failure: a report of the American College of Cardiology/American Heart Association Task Force on Clinical Practice Guidelines and the Heart Failure Society of America. J Am Coll Cardiol. 2017;70(6):776-803. doi:10.1016/j.jacc.2017.04.025
22. Packer M. Compelling first-line drug and device therapies for the prevention of sudden death in patients with chronic heart failure and a reduced ejection fraction who are candidates for an implantable cardioverter-defibrillator. Circ Arrhythm Electrophysiol. 2019;12(6): e007430. doi:10.1161/CIRCEP.119.007430

23. Lund LH, Svennblad B, Melhus H, Hallberg P, Dahlstrom U, Edner M. Association of spironolactone use with all-cause mortality in heart failure: a propensity scored cohort study. Circ Heart Fail. 2013;6(2):174-183. doi:10.1161/CIRCHEARTFAILURE.112.000115

24. Georgianos PI, Vaios V, Eleftheriadis T, Zebekakis P, Liakopoulos V. Mineralocorticoid antagonists in ESRD: an overview of clinical trial evidence. Curr Vasc Pharmacol. 2017;15(6):599-606. doi:10.2174/ 1570161115666170201113817

25. Jackson LD, Little J, Kung E, Williams EM, Siemiatkowska K, Plowman S. Safe medication swallowing in Dysphagia: a collaborative improvement project. Healthc $Q$. 2008;11(3Spec No):110-116. doi:10.12927/hcq. 2008.19660

26. Strachan I, Greener M. Medication-related swallowing difficulties may be more common than we realise. Pharm Pract. 2005;15(9):411-414.

27. Stegemann S, Gosch M, Breitkreutz J. Swallowing dysfunction and dysphagia is an unrecognized challenge for oral drug therapy. Int J Pharm. 2012;430(1-2):197-206. doi:10.1016/j.ijpharm.2012.04.022

28. Shen L, Jhund PS, Petrie MC, et al. Declining risk of sudden death in heart failure. $N$ Engl J Med. 2017;377(1):41-51. doi:10.1056/ NEJMoa1609758

29. Ferreira JP, Mentz RJ, Pizard A, Pitt B, Zannad F. Tailoring mineralocorticoid receptor antagonist therapy in heart failure patients: are we moving towards a personalized approach? Eur J Heart Fail. 2017;19(8):974-986. doi:10.1002/ejhf.2017.19.issue-8
Vascular Health and Risk Management

\section{Publish your work in this journal}

Vascular Health and Risk Management is an international, peerreviewed journal of therapeutics and risk management, focusing on concise rapid reporting of clinical studies on the processes involved in the maintenance of vascular health; the monitoring, prevention and treatment of vascular disease and its sequelae; and the involvement of metabolic disorders, particularly diabetes. This journal is indexed on PubMed Central and MedLine. The manuscript management system is completely online and includes a very quick and fair peerreview system, which is all easy to use. Visit http://www.dovepress. com/testimonials.php to read real quotes from published authors. 